\title{
Valor Diagnóstico da Tomografia por Emissão de Pósitrons / Tomografia Computadorizada (PET-CT) com Flúor-18 Fluordeoxiglicose (FDG-18F) em Pacientes com Carcinoma Diferenciado da Tireóide, Níveis Séricos de Tireoglobulina Elevados e Pesquisa de Corpo Inteiro com lodo Negativa
}

\begin{abstract}
RESUMO
Objetivo: Avaliar o papel da PET-CT com FDG-18F na detecção de recidiva e/ou metástase de carcinoma diferenciado da tireóide (CDT) em pacientes com níveis elevados de tireoglobulina (TG) e $\mathrm{PCl}$ negativa. Pacientes e método: Os achados da PET-CT de 25 pacientes foram comparados com a avaliação histopatológica e os métodos convencionais de imagem (MC). Resultados: A PET-CT foi positiva em 16 pacientes com resultado verdadeiro-positivo em 14 e falso-positivo em 2 casos (valor preditivo positivo 87,5\%). Nove pacientes tiveram PET-CT negativa; dois evoluíram com níveis indetectáveis de TG. Doença residual foi observada na $\mathrm{PCl}$ pós-dose terapêutica de uma paciente. Seis pacientes não apresentaram evidências de tumor durante o seguimento (média 16 meses). PETCT foi concordante com MC em 52\%, parcialmente concordante em $12 \%$ e discordante (6 falso-negativos e 3 falso-positivos dos $\mathrm{MC}$ ) em $36 \%$. Foi observada uma tendência de aumento da proporção de PET-CT positiva com o aumento de TG. Conclusão: A PET-CT com FDG-18F é útil na detecção de recidiva e/ou metástases de CDT com níveis de TG elevados mas $\mathrm{PCl}$ negativa. Apresenta alto valor preditivo positivo e é superior aos $\mathrm{MC}$, sendo mais efetiva quanto maior o nível de TG. (Arq Bras Endocrinol Metab 2007;51/4:581-586)
\end{abstract}

Descritores: Glândula tireóide; PET; Carcinoma; Tireoglobulina; Radioisótopos do iodo

\section{ABSTRACT}

Diagnostic Value of Positron Emission Tomography / Computed Tomography with Fluorine-18 Fluordeoxyglucose in Patients with Differentiated Thyroid Gland Carcinoma, High Thyroglobulin Serum Levels and Negative lodine Whole Body Scan.

Purpose: To evaluate the role of PET-CT with FDG- $18 \mathrm{~F}$ in the detection of recurrence and/or metastasis of differentiated thyroid carcinoma (DTC) in patients with elevated levels of thyroglobulin (TG) and negative whole body scan (WBS). Patients and method: PET-CT findings of 25 patients were compared to histopathology evaluation and conventional imaging (CI). Results: PET-CT scan was positive in 16 patients finding 14 true-positive and 2 false-positive cases (positive predictive value $87.5 \%$ ). Nine patients had negative PET-CT; two had decrease of TG to undetectable levels. One patient had residual disease detected by post-therapeutic WBS. Six patients had no evidence of tumor during follow-up (mean time 16 months). PET-CT was concordant with $\mathrm{Cl}$ in $52 \%$, partially concordant in $12 \%$ and discordant in $36 \%$ (6 false-negatives and 3 false-positive of $\mathrm{Cl}$ ). We observed a tendency of increasing proportion of positive PET-CT with increasing TG. Conclusion: PET-CT scan with FDG- ${ }^{18} \mathrm{~F}$ is useful in the detection of recurrence and/or metastases of DTC with high TG levels but negative WBS. It presents elevated positive predictive value and is superior to $\mathrm{Cl}$ being more effective as higher the serum TG levels. (Arq Bras Endocrinol Metab 2007;51/4:581-586)

Keywords: Thyroid gland; PET scan; Carcinoma; Thyroglobulin; lodine radioisotopes

\section{artigo original}

\author{
LILIAN YURI ItAYA YAMAGA \\ MARCELO LIVORSI DA CUNHA \\ JAIRO WAGNER \\ ANNELISE FISCHER THOM \\ MaURo Miguel DaNiel \\ MARCElo B. de Gusmão FunarI
}

Departamento de Imagem, Hospital Israelita Albert Einstein, São Paulo, SP.

Recebido em 26/12/05

Revisado em 17/03/06

Aceito em 20/03/07 
A DOSAGEM SÉRICA DE TIREOGLOBULINA (TG) e a pesquisa de corpo inteiro (PCI) com iodo-131 (131I) ou iodo-123 (123I) são métodos bem estabelecidos para o seguimento de pacientes após o tratamento do carcinoma diferenciado da tireóide (CDT) (1). A dosagem de TG obtida por ensaio imunorradiométrico é o marcador mais sensível e específico de CDT. Concentração sérica elevada de TG geralmente indica recidiva ou doença metastática e está freqüentemente associada a resultado anormal da PCI $(1,2)$.

Entretanto, alguns pacientes têm doença metastática ou recidiva tumoral (com conseqüente aumento dos níveis de TG) mas a PCI é negativa, mesmo quando esta é realizada na vigência de dose terapêutica de ${ }^{131}$ I. A sensibilidade varia de 45 a $75 \%$, ocorrendo resultados falso-negativos por micrometástases ou devido à perda da capacidade de a célula neoplásica concentrar iodo. Este fato decorre da desdiferenciação das células tumorais que perdem a capacidade de concentrar iodo, levando a resultado falso-negativo da PCI $(3,4)$.

Inúmeras investigações vêm demonstrando a importância da tomografia por emissão de pósitron (PET) com flúor-18 fluorodesoxiglicose (FDG-18F) na detecção de recidiva local ou metástase de CDT. Este método é particularmente útil na detecção das células tumorais menos diferenciadas que apresentam elevada taxa de consumo de glicose e não captam iodo (5-7). $\mathrm{O}$ recente advento do equipamento híbrido de PET e CT oferece alta precisão na localização anatômica das lesões neoplásicas funcionalmente alteradas, pois avalia tanto o metabolismo quanto a anatomia do corpo inteiro num único exame. As imagens de co-registro PET-CT são particularmente úteis na região cervical, onde algumas estruturas normais com captação fisiológica da glicose marcada (tecido linfóide da naso/orofaringe, atividade muscular e da gordura marrom) podem dificultar a detecção da lesão hipermetabólica tumoral.

O presente estudo avaliou o papel da PET-CT com FDG-18F no seguimento de pacientes com carcinoma da tireóide com aumento dos níveis de TG e PCI negativa.

\section{PACIENTES E MÉTODO}

Foram avaliados 25 pacientes ( 17 do sexo feminino, 8 do sexo masculino, idades entre 14 e 74 anos, média 41,6 anos) com CDT confirmado por estudo histopatológico $(22 \mathrm{com}$ carcinoma papilífero, 3 com carcinoma folicular, incluindo um com carcinoma de células de Hürthle). Todos os casos tinham sido previamente submetidos à tireoidectomia total seguida de uma ou mais doses terapêuticas de 131I. Todos apresentavam níveis séricos elevados de TG e resultado negativo da PCI na ocasião da PET-CT com FDG-18F. Foram previamente excluídos da casuística os pacientes com dosagem positiva de anticorpos anti-TG.

A concentração de TG no soro foi determinada por ensaio imunorradiométrico (kit comercial Dynotest Brahms $^{\circledR}$, Alemanha, com sensibilidade funcional de 0,2 $\mathrm{ng} / \mathrm{mL}$ ). Foram considerados níveis elevados de TG quando os valores foram superiores a $5 \mathrm{ng} / \mathrm{mL}$ durante estímulo de TSH (TSH superior a $30 \mathrm{mUI} / \mathrm{L}$ ) ou superiores a $1 \mathrm{ng} / \mathrm{mL}$ durante supressão de TSH. As dosagens séricas de TSH foram obtidas por quimioluminescência (kit comercial Bayer ${ }^{\circledR}$, sensibilidade funcional $0,01 \mathrm{mUI} / \mathrm{mL}$ ).

Os estudos de PET-CT foram realizados com os pacientes em jejum de, no mínimo, 4 horas e estado normoglicêmico. Vinte e dois pacientes fizeram o exame sob estimulação de TSH (através da suspensão da reposição hormonal por cerca de 30 dias, em 15 casos, ou na vigência de TSH recombinante, em 7 casos).

As imagens da PET-CT foram obtidas em equipamento dedicado de PET Discovery ST GE ${ }^{\circledR}$ acoplado à CT multislice com 8 fileiras de detectores (GE modelo LIGHTSPEED), 60 minutos após a administração e.v. de 10 a $15 \mathrm{mCi}(370-555 \mathrm{MBq})$ de FDG- $^{-18} \mathrm{~F}$.

Os cortes axiais, coronais e sagitais das imagens de fusão da PET com CT foram analisados por dois médicos experientes. Os casos de discordância de interpretação foram resolvidos por consenso. Área de acúmulo anômalo de FDG${ }^{18} \mathrm{~F}$ foi definida como captação focal maior do que o tecido adjacente, sem atividade semelhante na região contralateral, na projeção de uma estrutura anatômica identificável nas imagens de CT.

Os achados da PET-CT foram correlacionados com a análise histopatológica de 14 pacientes que foram, posteriormente, submetidos a cirurgia ou biópsia para tratamento de recidiva tumoral.

Os resultados da PET-CT também foram comparados com os observados nos métodos convencionais de imagem (MC), incluindo US do pescoço, CT ou RM do pescoço, tórax e coluna lombar.

\section{Análise estatística}

Foi utilizado o teste de tendência linear de Cochran-Armitage para verificar a correlação entre o nível de TG e o resultado da PET-CT. Valores de p menores que 0,05 foram considerados estatisticamente significantes e valores entre 0,05 e 0,10 , como tendência linear marginalmente significante $(8)$.

\section{RESULTADOS}

PET-CT positiva: A PET-CT detectou uma ou mais áreas de aumento do metabolismo de FDG-18F em 16 pacientes, conforme mostra a tabela 1 . As lesões hipermetabólicas se localizaram no leito tireoidiano $(\mathrm{n}=5$ casos $)$, linfonodos cervicais e mediastinais $(\mathrm{n}=$ $12)$, esqueleto $(n=1)$ e pulmões $(n=2)$. Catorze dos 
Tabela 1. Resultados dos métodos convencionais de imagem, da PET-CT com FDG-18F e do estudo histopatológico dos 25 pacientes com carcinoma diferenciado da tireóide.

\begin{tabular}{|c|c|c|c|c|c|}
\hline Pac. & Sex/id & Histol. & Imagem Convencional & PET-CT & Histopatol. \\
\hline 1 & $\mathrm{~F} / 45$ & Pap & Leito tireoidiano (CT) & Leito tireoidiano & Positivo \\
\hline 2 & $F / 38$ & Pap & LN jugular D (US, RM) & LN jugular D & Positivo \\
\hline 3 & $\mathrm{M} / 67$ & Pap & LNM cervical D (US) & $\begin{array}{l}\text { Leito tireoidiano } \\
\text { LNM cervicais D }\end{array}$ & Positivo \\
\hline 4 & $\mathrm{~F} / 26$ & Pap & LNM retrofaringea (CT) & LNM retrofaringea & Positivo \\
\hline 5 & $\mathrm{M} / 56$ & Pap & 3 LN cervicais (US) & LNM paratraqueal D & Positivo \\
\hline 6 & $\mathrm{~F} / 70$ & Fol & $\mathrm{L}-2(\mathrm{CT})$ & $\mathrm{L}-2$ & Positivo \\
\hline 7 & $\mathrm{~F} / 41$ & Pap & Negativo & $\begin{array}{l}\text { Leito tireoidiano } \\
\text { LN júgulo-carotídeo } D\end{array}$ & Positivo \\
\hline 8 & $F / 31$ & Pap & Negativo & Nódulo paratraqueal & Positivo \\
\hline 9 & $\mathrm{M} / 32$ & Pap & Negativo & LN mediastinais & Positivo \\
\hline 10 & $\mathrm{~F} / 51$ & Pap & Negativo & Leito tireoidiano & Positivo \\
\hline 11 & $F / 48$ & Pap & Negativo & Leito tireoidiano & Positivo \\
\hline 12 & $\mathrm{~F} / 27$ & Pap & Negativo & LN paratraqueal E & Positivo \\
\hline 13 & $\mathrm{M} / 56$ & Pap & Nódulos pulmonares (CT) & $\begin{array}{l}\text { LN paratraqueal D } \\
\text { Múltiplos nódulos } \\
\text { pulmonares }\end{array}$ & \\
\hline 14 & $\mathrm{M} / 74$ & Hurt & $\begin{array}{c}\text { LNM cervicais } \mathrm{E} \\
\text { Nódulos pulmonares (CT) }\end{array}$ & $\begin{array}{l}\text { LNM cervical E } \\
\text { Nódulos pulmonares }\end{array}$ & $\begin{array}{l}\text { Não realizado } \\
\text { Não realizado }\end{array}$ \\
\hline 15 & $\mathrm{~F} / 14$ & Pap & LN cervical E (US) & LN cervical E & Inflamatório \\
\hline 16 & $\mathrm{~F} / 40$ & Pap & LN cervical D (US) & LN cervical D & Inflamatório \\
\hline 17 & $\mathrm{~F} / 43$ & Pap & Negativo & Negativo & Não realizado \# \\
\hline 18 & $\mathrm{M} / 35$ & Pap & Negativo & Negativo & Não realizado \\
\hline 19 & $\mathrm{M} / 40$ & Pap & Negativo & Negativo & Não realizado \\
\hline 20 & $\mathrm{~F} / 28$ & Fol & Negativo & Negativo & Não realizado \\
\hline 21 & $\mathrm{~F} / 16$ & Pap & Negativo & Negativo & Não realizado \\
\hline 22 & $\mathrm{~F} / 54$ & Pap & Negativo & Negativo & Não realizado \\
\hline 23 & $\mathrm{M} / 22$ & Pap & LN cervical (US) & Negativo & Não realizado \# \\
\hline 24 & $\mathrm{~F} / 40$ & Pap & LN cervicais $(\mathrm{CT})$ & Negativo & Não realizado \\
\hline 25 & $\mathrm{~F} / 45$ & Pap & 3 LN cervicais (US) & Negativo & Não realizado * \\
\hline
\end{tabular}

Pac.: Identificação do paciente, Sex/ld: sexo e idade do paciente, Imagem convencional: métodos convencionais de imagem, Fol: carcinoma folicular, Hurt: carcinoma de células de Hürthle, LN: Linfonodo, LNM: Linfonodomegalia, Pap: carcinoma papilífero, Histopatol: Estudo histopatológico, Hist: tipo histológico, L-2: segunda vértebra lombar.

\# Tg indetectável no seguimento

* PCl pós dose terapêutica positiva no leito tireoidiano

16 pacientes com PET-CT positiva realizaram cirurgia ou biópsia das alterações evidenciadas na PET-CT, obtendo confirmação histopatológica de lesão tumoral em 12 (resultado positivo-verdadeiro). A figura 1 (caso 3) exemplifica um resultado verdadeiro-positivo de PET-CT com lesão no leito tireoidiano e PCI negativa. Dois casos tiveram resultado falso-positivo da PET-CT com captação de FDG-18F em linfonodo cervical, cuja biópsia mostrou alterações inflamatórias. Dois outros casos com múltiplas lesões ganglionares e pulmonares evidenciadas na PET-CT tiveram alterações correspondentes no estudo dedicado de CT na maioria das áreas captantes (caso 13, figura 2). Ambos os pacientes não foram operados devido ao comprometimento extenso pela doença.
PET-CT negativa: Padrão normal de distribuição do FDG-18F (PET-CT negativa) foi observado em 9 casos. Dois destes pacientes apresentaram diminuição espontânea da concentração sérica de TG para níveis indetectáveis durante o seguimento. Uma paciente fez tratamento com altas doses de ${ }^{131}$ I, evidenciando-se captação no leito tireoidiano na PCI obtida na vigência da dose terapêutica. Os outros 6 pacientes foram seguidos clinicamente, não apresentando evidência clínica ou pelos métodos convencionais de imagem de lesão tumoral até o término do presente estudo (tempo médio de seguimento de 16 meses). Não foram calculadas a sensibilidade e a especificidade da PET-CT devido à ausência de um teste padrão ouro indicativo de ausência de doença. 

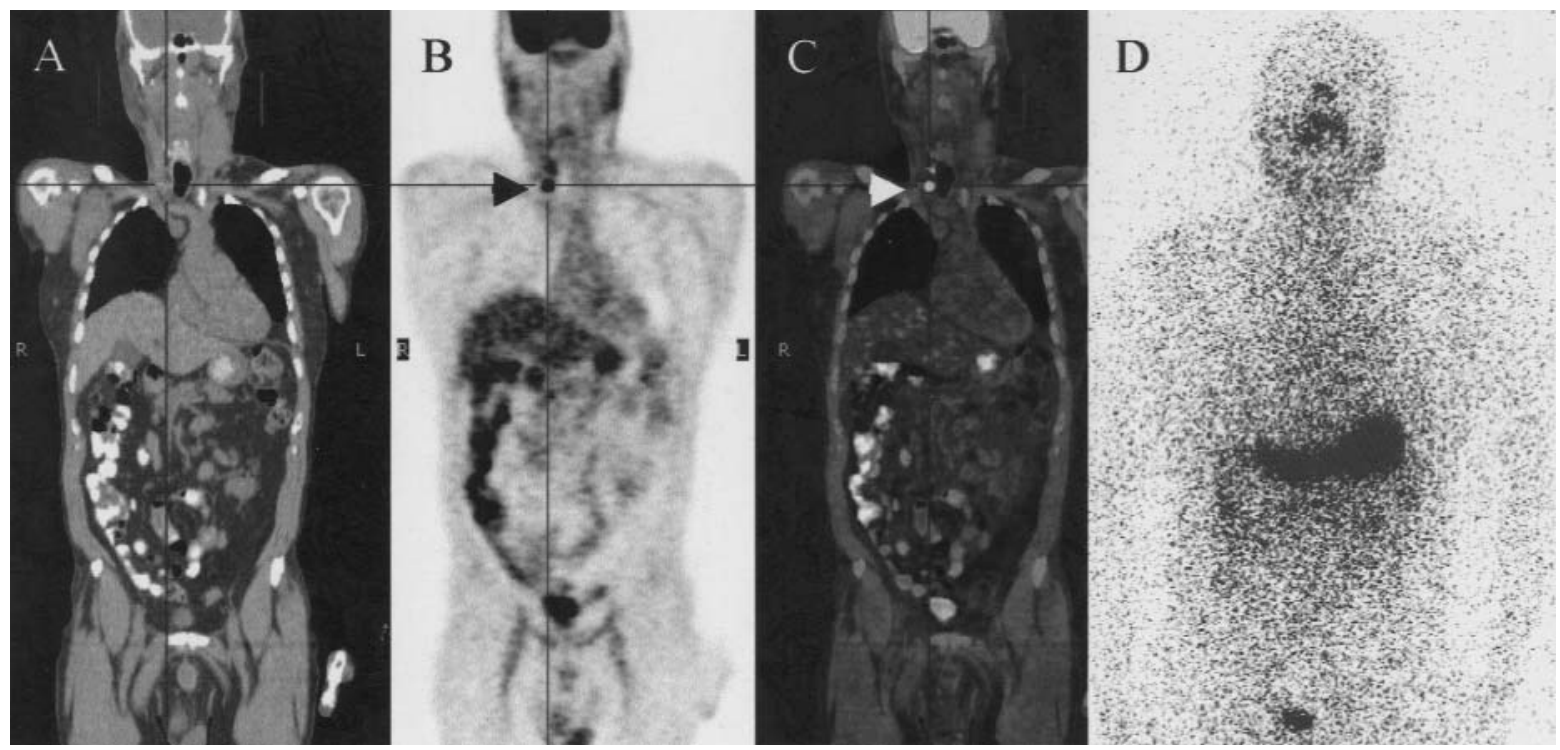

Figura 1. Paciente $n^{\circ}$ 3: Paciente masculino de 67 anos com carcinoma papilífero da tireóide. Cortes coronais da CT (A), PET com FDG-18 F (B) e imagem de fusão PET-CT (C) revelam duas áreas metabolicamente ativas no leito tireoidiano à direita (seta). $\mathrm{PCl}$ é negativa (D).
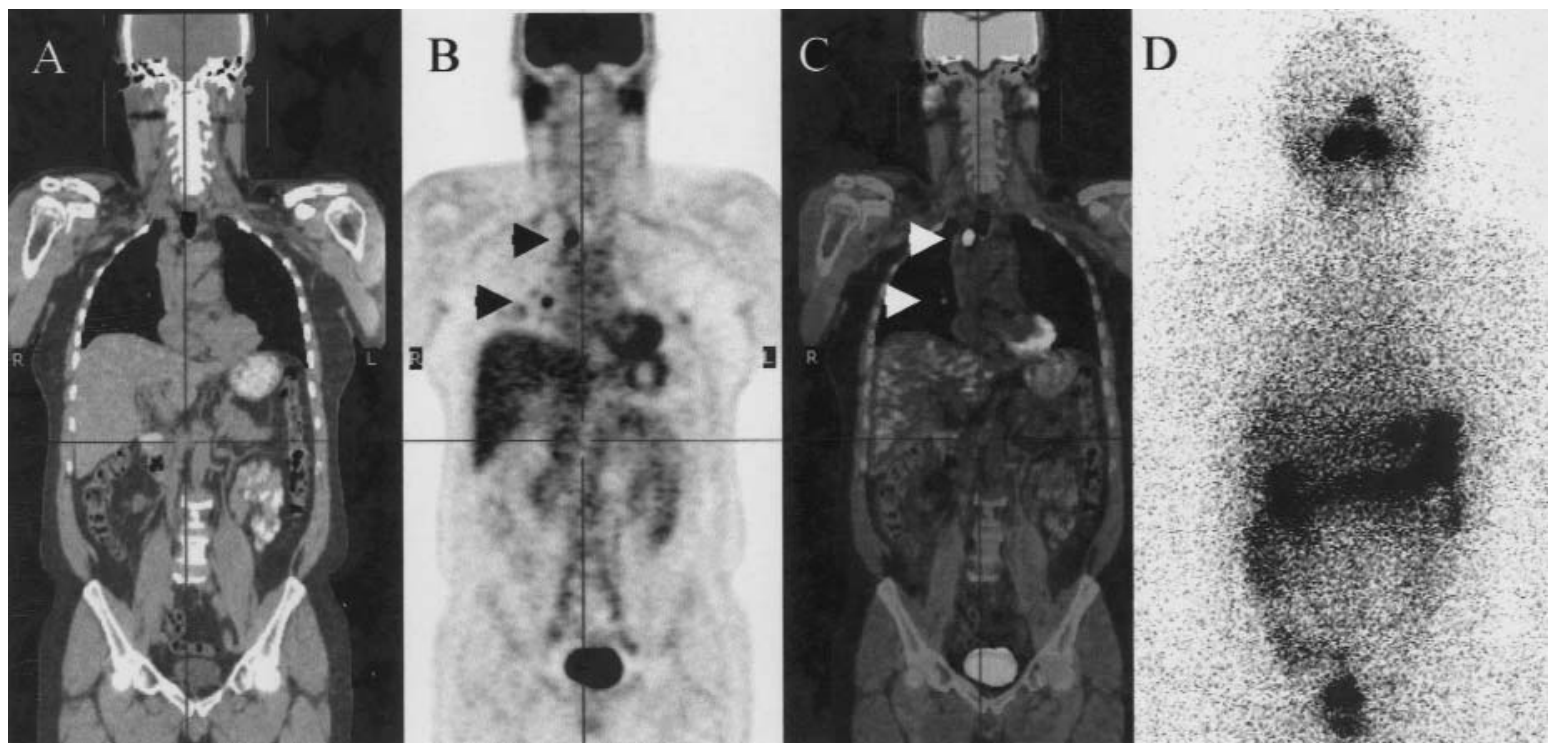

Figura 2. Paciente $n^{\circ}$ 13: Paciente masculino de 56 anos com carcinoma papilífero da tireóide. Cortes coronais da CT (A), PET com FDG-18F (B) e imagem de fusão PET-CT (C) demonstram metástases de carcinoma da tireóide em um linfonodo paratraqueal alto à direita e em ambos os pulmões (setas). $\mathrm{A} \mathrm{PCl} \mathrm{é} \mathrm{negativa} \mathrm{(D).}$

PET-CT e os métodos convencionais de imagem (MC): Os resultados da PET-CT foram concordantes com MC em 13 dos 25 pacientes (52\%). Em cinco pacientes, a PET-CT confirmou o número e a localização das lesões previamente suspeitadas pelos MC. Dois pacientes apresentaram lesões captantes na PET-CT que não tinham sido suspeitadas clinicamente no mediastino e na coluna lombar, respectivamente, que foram confirmadas posteriormente pelos MC. Seis dos 13 pacientes tiveram resultado negativo tanto na PET-CT quanto nos MC.

PET-CT e MC foram parcialmente concordantes em 3 pacientes (12\%) à custa de maior número de lesões detectadas na PET-CT do que indicavam os 
exames anatômicos (resultado falso-negativo de MC no leito tireoidiano e em linfonodos cervicais).

Resultados discordantes ocorreram em 9 dos 25 pacientes $(36 \%)$ com resultados falso-negativos e falsopositivos dos MC em 6 e 3 indivíduos (linfonodos cervicais observados no US não captantes na PET-CT, cujo seguimento clínico excluiu malignidade), respectivamente.

Níveis séricos de TG: A análise do efeito da concentração sérica de TG sobre o resultado da PET-CT mostrou que houve uma tendência linear marginalmente significativa $(\mathrm{p}=0,0794)$ de aumento da proporção de PET-CT positiva com o aumento dos níveis de TG.

\section{DISCUSSÃO}

O presente estudo demonstrou o valor da PET com FDG- ${ }^{18} \mathrm{~F}$, que localizou a recidiva e/ou metástases de CDT em 87,5\% dos pacientes com níveis de TG elevados mas PCI negativa. Os resultados aqui apresentados confirmam os achados da literatura que estão resumidos na tabela 2 . Um estudo multicêntrico que incluiu 222 pacientes com CDT evidenciou sensibilidade global da PET com FDG- $^{-18} \mathrm{~F}$ de $75 \%$, mas a sensibilidade aumentou para $87,5 \%$ quando se incluiu apenas o subgrupo dos 65 pacientes com PCI negativa (9). Segundo Reske e Kotzeike, a sensibilidade e a especificidade da PET com FGD-18F na detecção de metástases iodonegativas e TG aumentada é de 85 a $94 \%$ e 90 a $95 \%$, respectivamente (10).

A PET-CT foi negativa em 7 pacientes da presente casuística que evoluíram sem evidências clínicas ou de imagem convencional de tumor, mas cursaram com TG persistentemente elevada durante o seguimento. Uma possível explicação seria o pequeno tamanho das lesões associado ao baixo grau de captação de FDG-18F. Esta hipótese é reforçada pelos achados de outros investigadores, que mostraram resultado falso-negativo da PET em doença mínima localizada em linfonodos cervicais $(11,12)$.
Dois pacientes da presente casuística apresentaram captação anômala de FDG-18 F em linfonodo cervical por processo inflamatório, que é uma das causas mais freqüentes de resultado falso-positivo da PET.

$O$ resultado da PET é influenciado pelo nível sérico de TG, sendo mais efetivo quando a concentração de TG é superior a $10 \mathrm{ng} / \mathrm{mL}$. Ressalvado o pequeno número de casos, os nossos resultados são concordantes com os achados da literatura. A explicação para este fato é que a produção de TG depende do número de células tumorais. Quanto maior o tamanho do tumor, mais elevado é o nível sérico de TG e, portanto, maior a chance de esta lesão ser detectada pela PET (5).

A sensibilidade do método aumenta quando é feita na vigência de estimulação de TSH, seja através da suspensão da reposição hormonal, seja pelo uso do TSH recombinante. O TSH estimula o sistema de transporte de glicose na membrana celular aumentando o grau de captação de FDG-18F no tumor $(13,14)$. No presente estudo, a relação entre o nível de TSH e o resultado da PET-CT não foi avaliado, pois 22 dos 25 pacientes realizaram a pesquisa sob estímulo do TSH.

Outro fator que influi nos resultados da PET está relacionado ao equipamento utilizado. Segundo Lind e cols., que avaliaram 27 pacientes com CDT, a PET-CT teve maior acurácia que a PET sem fusão ou a CT separadamente em $67 \%$ dos casos e mudou a conduta terapêutica de $17 \%$ dos casos (4).

Nossos resultados demonstram, ainda, que a PET-CT é superior aos métodos convencionais de imagem com maior porcentagem de resultados verdadeiropositivos e menor número de resultados falso-negativos em comparação com o US, CT e/ou RM. As vantagens da PET-CT sobre os métodos anatômicos de imagem incluem diferenciar recidiva tumoral de alteração póscirúrgica, detecção de metástases em linfonodos de tamanho normal e permitir o rastreamento do corpo inteiro num só exame (9).

Dentre os métodos de imagem, o US do pescoço é o método de escolha para a detecção de recidiva local e de metástases em linfonodos cervicais, com

Tabela 2. Resultados da literatura e do presente estudo sobre o valor da PET-CT com FDG-18F na avaliação de pacientes com carcinoma diferenciado da tireóide, níveis elevados de TG mas PCI negativa.

\begin{tabular}{lcccccc}
\hline Autor (ref) & N pac & Sens (\%) & Esp (\%) & VPP (\%) & VPN (\%) & Confirm. \\
Wang, 1999 (13) & 18 & 71 & NA & 92 & NA & I/H \\
Grunwald, 1999 (9) & 65 & 87,5 & 100 & 100 & 83,3 & $1 / H$ \\
Schlüter, 2001 (4) & 48 & NA & NA & 83 & 25 & $1 / \mathrm{H}$ \\
Nahas, 2005 (14) & 33 & 66 & 100 & 100 & 27 & $1 / \mathrm{H}$ \\
Presente estudo & 25 & NA & NA & 87,5 & NA & I/H
\end{tabular}

Ref: referência bibliográfica, $\mathrm{N}$ pac: número de pacientes com TG elevada e $\mathrm{PCl}$ negativa, Sens: sensibilidade, Esp: especificidade, VPP: valor preditivo positivo, VPN: valor preditivo negativo, Confirm.: confirmação, l/H: método de imagem e achado histológico, NA: não disponível e/ou não aplicável. 
sensibilidade de 70 a 95\%. Entretanto, a especificidade do US é de apenas 70\% (15-17). A biópsia aspirativa guiada por US e dosagem de TG dos linfonodos constituem um grande avanço no diagnóstico das metástases cervicais de CDT. Além disso, a avaliação pelo US não exclui metástases a distância. Doença locorregional pode ocorrer concomitantemente a metástases a distância em até 18\% dos casos, e metástases a distância sem acometimento locorregional ocorrem em até 11\% (4).

Outros MC como CT e RM também apresentam limitações na avaliação da recidiva local, metástases locorregionais e a distância devido à interferência das alterações pós-cirúrgicas e limitação do seu campo de análise (17).

Em resumo, o presente estudo confirma a utilidade da PET-CT com FDG-18F na detecção de recidiva e/ou metástases de carcinoma bem diferenciado da tireóide nos casos com níveis séricos elevados de TG e PCI negativa. Apresenta alto valor preditivo positivo, porém o resultado negativo não exclui doença residual. É superior aos métodos anatômicos de imagem na localização das lesões tumorais e é mais efetiva quanto maior o nível de TG sérico. Investigações futuras com maior casuística deverão confirmar os achados do presente estudo.

\section{AGRADECIMENTOS}

Agradecemos aos Drs. Miguel José Francisco Neto e Fernanda Chiarot Flores Fleming, por sua cooperação na elaboração do presente manuscrito.

\section{REFERÊNCIAS}

1. Schlumberger MJ. Papillary and follicular carcinoma. N Engl J Med 1998;338:297-306.

2. Pacini F, Lari R, Mazzeo S, Grasso L, Taddei D, Pinchera A. Diagnostic value of single serum thyroglobulin determination on and off thyroid suppressive therapy in the follow-up of patient with differentiated thyroid cancer. Clin Endocrinol 1985;23:405-8.

3. Schlumberger M, Arcangioli O, Piekarski JD, Tubiana M, Parmentier C. Detection and treatment of lung metastases of differentiated thyroid carcinoma in patients with normal chest X-rays. J Nucl Med 1988;29:1790-4.

4. Lind $\mathrm{P}$, Kohlfürst $\mathrm{S}$. Respective roles of thyroglobulin, radioiodine imaging and positron emission tomography in the assessment of thyroid cancer. Semin Nucl Med 2006;36:194-205.
5. Schlüter B, Bohuslavizki KH, Beyer W, Plotkin M, Buchert R, Clausen M. Impact of FDG PET on patients with differentiated thyroid cancer who present with elevated thyroglobulin and negative 131/ scan. J Nucl Med 2001;42:71-6.

6. Helal BO, Merlet P, Toubert M, Franc B, Schvarts C, GauthierKoelesnikov, et al. Clinical impact of 18 F-FDG PET in thyroid carcinoma patients with elevated thyroglobulin levels and negative 131 scanning results after therapy. J Nucl Med 2001:42:1464-9.

7. Grunwald F, Schomburg A, Bender H, Klemm E, Menzel C, Bultmann $T$, et al. Fluorine-18 fluordeoxyglucose positron emission tomography in the follow-up of differentiated thyroid cancer. Eur J Nucl Med 1996;23:312-9.

8. Armitage $P$, Berry G. Statistical methods in medical research. 2nd ed. Oxford: Blackwell, 1987.

9. Grunwald F, Kaliche T, Feine U, Lietzenmayer R, Scheidhauer $\mathrm{K}$, Dietle $\mathrm{M}$, et al. Fluorine18-fluordesoxyglucose positron emission tomography in thyroid cancer: results of a multicentre study. Eur J Nucl Med 1999;26:1547-52.

10. Reske SN, Kotzerke J. FDG PET for clinical use. Results of the $3^{\text {rd }}$ German Interdisciplinary Consensus Conference. Eur J Nucl Med 2001;28:1707-23.

11. Wang W, Macapinlac H, Larson SM, Yeh SDJ, Akhurst T, Finn $\mathrm{RD}$, et al. [18F]-2-fluor-2-deoxy-D-glucose positron emission tomography localizes residual thyroid cancer in patients with negative diagnostic 131 . whole body scans and elevated serum thyroglobulin levels. J Clin Endocrinol Metab 1999;84:2291-302.

12. Nahas Z, Goldenberg D, Fakhry C, Ewertz M, Zeiger M, Ladenson PW, et al. The role of positron emission tomography/computated tomography in the management of recurrent papillary thyroid carcinoma. Laryngoscope 2005; 115:237-43.

13. Moog F, Linke R, Manthey N, Tiling R, Knesewitsch P, Tatsch $K$, et al. Influence of thyroid-stimulating hormone levels on uptake of FDG in recurrence and metastatic differentiated thyroid carcinoma. J Nucl Med 2000;41:1989-95.

14. Petrick T, Borner AR, Otto D, Hofmann M, Knapp WH. Influence of rhTSH on $\left({ }^{18} \mathrm{~F}\right)$ fluorodeoxyglucose uptake by differentiated thyroid carcinoma. Eur J Nucl Med 2002;29:641-7.

15. Frasoldati A, Pesenti M, Gallo M, Caroggio A, Salvo D, Valcavi R. Diagnosis of neck recurrences in patients with differentiated thyroid carcinoma. Cancer 2003;97:90-6.

16. Pacini F, Molinaro F, Castagna MG, Agate L, Elisei R, Cecarelli $C$, et al. Recombinant human thyrotropin stimulated serum thyroglobulin combined with neck ultrasonography has the highest sensitivity in monitoring differentiated thyroid carcinoma. J Clin Endocrinol Metab 2003;88:3668-73.

17. Stokkel M, Duchateau C, Dragoiescu C. The value of FDG-PET in the follow-up of differentiated thyroid cancer: a review of the literature. Q J Nucl Med Mol Imaging 2006;50:78-87.

Endereço para correspondência:

Lilian Yuri Itaya Yamaga

Hospital Israelita Albert Einstein 701

05651-901 São Paulo, SP

E-mail: itaya@einstein.br 